\title{
Line Defect-Layered EBG Waveguides in Dielectric Substrates
}

\author{
Hassna Ouassal $\mathbb{D}^{1},{ }^{1}$ Jafar Shaker, ${ }^{2}$ Langis Roy, ${ }^{3}$ Khelifa Hettak, ${ }^{2}$ and Reza Chaharmir ${ }^{2}$ \\ ${ }^{1}$ Department of Electronics, Carleton University, Ottawa, ON, Canada \\ ${ }^{2}$ Communications Research Centre, Ottawa, ON, Canada \\ ${ }^{3}$ University of Ontario Institute of Technology, Oshawa, ON, Canada
}

Correspondence should be addressed to Hassna Ouassal; houassal@doe.carleton.ca

Received 24 February 2018; Accepted 23 April 2018; Published 4 June 2018

Academic Editor: Giorgio Montisci

Copyright (C) 2018 Hassna Ouassal et al. This is an open access article distributed under the Creative Commons Attribution License, which permits unrestricted use, distribution, and reproduction in any medium, provided the original work is properly cited.

\begin{abstract}
A dielectric-based multilayer structure composed of U-shaped rings (ML-UR) is used to develop a class of novel electromagnetic band gap (EBG) slab waveguide. The structure has two band gaps that narrow down as dielectric constant is increased. The EBG slab waveguide is created by embedding a single-layer line defect inside the 3D crystal of the EBG slab guide. Unlike our previously published foam-based EBG structure, the use of dielectric spacer in the EBG waveguides offers significant advantages in terms of overall size, structure reliability, and design flexibility. The waveguide structures reported in this paper are designed to operate at X-band $(8-12 \mathrm{GHz})$ while being fed by coplanar-slotline transitions. Prototypes were fabricated and characterized experimentally. The insertion loss decreases by decreasing the number of full lattices of ML-UR surrounding the channels. The proposed waveguide has potential in microwave components such as directional couplers, phase shifters, and antenna array feeding networks.
\end{abstract}

\section{Introduction}

Electromagnetic band gap (EBG) structures comprised of multilayers of U-shaped rings (ML-UR), shown in Figure 1(a), were first introduced in $[1,2]$ as an alternative EBG structure for novel microwave designs. This construction is of great interest due to its relatively simple fabrication technology (no via holes required), light weight, and compatibility with planar circuits for integration of active components. The structure presents a potential alternative as compared to the conventional photonic band gap composed of dielectric rods [3]. The lattice of ML-UR can be understood as a lattice of rods comprised of high-permeability material. Development of transmission lines using this approach paves the way for its wider application to a wide variety of microwave circuits and components. TE-polarized line defect waveguides (magnetic field is normal to the plane of the waveguide and electric field is in the plane) have been developed in foam $\left(\varepsilon_{r} \sim 1\right)$ [4] and dielectric $\left(\varepsilon_{r}=2.94\right)$ [5] media. The EBG waveguides in air (foam) have disadvantages including the lack of structural robustness and significant radiation losses for narrower width. In this paper, we extend the study that was presented in [5]. The propagation in dielectric-supported ML-UR is investigated and verified in modified versions of the waveguide of [5] for the first time. The dielectric material is necessary to achieve a rigid structure. It also permits strongly confined guided modes and less radiation at discontinuities. The loss in the proposed waveguide increases with width (number of rows). This is contrary to the behavior of the waveguide in air. Comparisons of the proposed structure with the other published waveguides are summarized in Table 1.

This paper presents characteristics and application of this dielectric-embedded ML-UR. Infinite and finite designs are introduced in Section 2, where the geometry of the EBG lattice and its theoretical characterization are presented. Section 3 focuses on the design of ML-UR slab and Section 4 deals with the design of the slab waveguide. Detailed waveguide characterization is presented in Section 5, while Section 6 gives concluding remarks. 


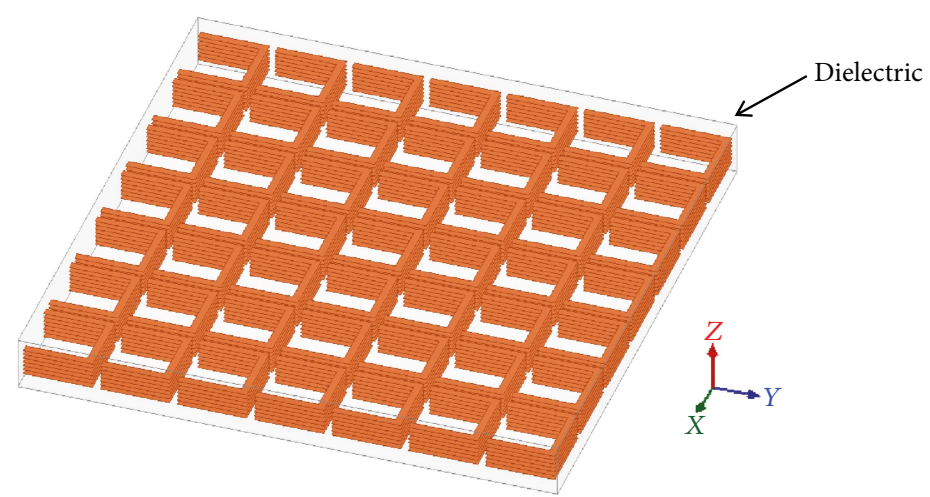

(a)

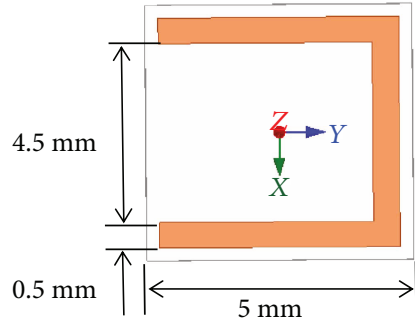

(b)

Figure 1: (a) Schematic of the ML-UR structure: 2D square array of stacked U-rings in a dielectric medium. (b) The unit cell of the infinite design.

TABLe 1: Comparison of the ML-UR waveguides.

\begin{tabular}{|c|c|c|c|c|c|c|}
\hline Ref & $\varepsilon_{r}$ & Freq $(\mathrm{GHz})$ & Number of rows & Channel length (periods) & Structure strength & Loss $\left(\mathrm{dB} / \lambda_{g}\right)$ \\
\hline \multirow{2}{*}{ [4] } & \multirow{2}{*}{$\sim 1$} & \multirow{2}{*}{15.3} & 3 & 4 & \multirow{2}{*}{ Low } & 4 \\
\hline & & & 6 & 4 & & 3.1 \\
\hline$[5]$ & \multirow{4}{*}{2.94} & \multirow{4}{*}{9.8} & 1 & 8 & \multirow{4}{*}{ High } & 1.09 \\
\hline \multirow{3}{*}{ This work } & & & 3 & 8 & & 1.8 \\
\hline & & & 3 & 4 & & 3.08 \\
\hline & & & 6 & 4 & & 5.1 \\
\hline
\end{tabular}

Number of rows refers to the layered rings beside the channel, Figure 8(a). Channel length refers to the number of periods along the channel, Figure $8(a) . \lambda_{g}$ is the guided wavelength at the indicated frequency of operation band.

\section{Study of the EBG ML-UR in Dielectric Media}

In this section, dependence of the band gap (BG) of the infinitely thick ML-UR on the host dielectric material $\varepsilon_{r}$ is presented. A relationship is established between the size of the BG and thickness of ML-UR slab for a given permittivity of the host material.

2.1. Infinite ML-UR Structure. The U-ring cell element geometry is shown in Figure 1(b). The ring is immersed in a dielectric medium to form a periodic in $x, y$, and $z$ directions. The $3 \mathrm{D}$ lattice is actually composed of layers of $2 \mathrm{D}$ arrangements of $\mathrm{U}$-rings that are populated along the $z$-axis.

Figure 2 presents the BG frequencies as a function of the permittivity of the host dielectric medium. Information on the calculation of the band gap can be found in $[1,4]$. It was observed that the band gap narrowed down and shifted to lower frequency band as the permittivity is increased. Furthermore, the ML-UR design exhibited a second BG that can be instrumental in the suppression of undesired harmonics.

\section{ML-UR Slab Design}

Having selected a dielectric constant $\varepsilon_{r}$ of 2.94 and exploiting the first band gap, we investigated the effect of slab thickness $d$ (i.e., finite ML-UR along $z$ ) on the width of the BG for the cases of 3 and 7 rings along $z$-axis, separated by $H=0.25 \mathrm{~mm}$

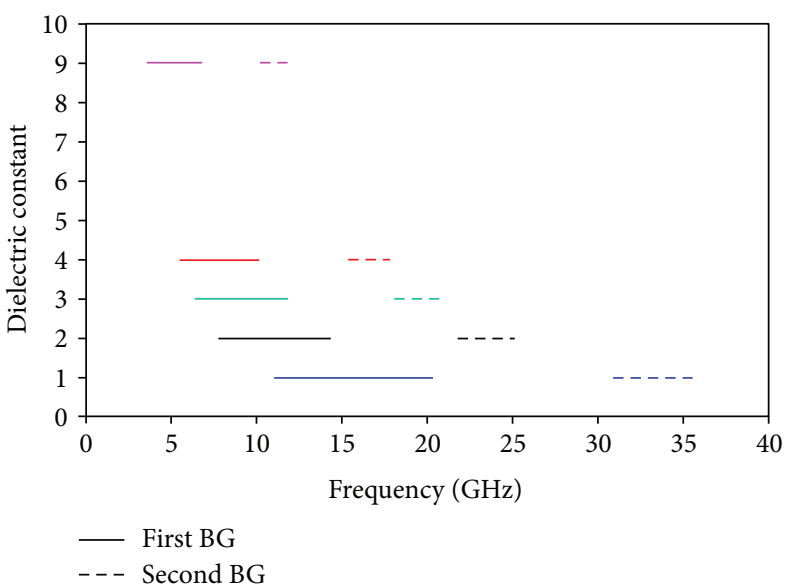

FIgURE 2: BG frequencies of the infinite ML-UR as a function of dielectric constant of the host material. The solid line is the first $\mathrm{BG}$ and the dashed line is the second $\mathrm{BG}$.

and $1 \mathrm{~mm}$ along the $z$-axis. The slab enables confinement of electromagnetic (EM) energy along $z$ while the band gap enables the field confinement in $x y$ plane. For a typical slab of thickness $d=3 \mathrm{~mm}$ and 7 rings with $0.25 \mathrm{~mm}$ spacing, Figure 3 shows the unit cell of the slab. Periodic boundary conditions were enforced on the sides and perfect magnetic conductor (PMCs) boundary conditions were imposed on 


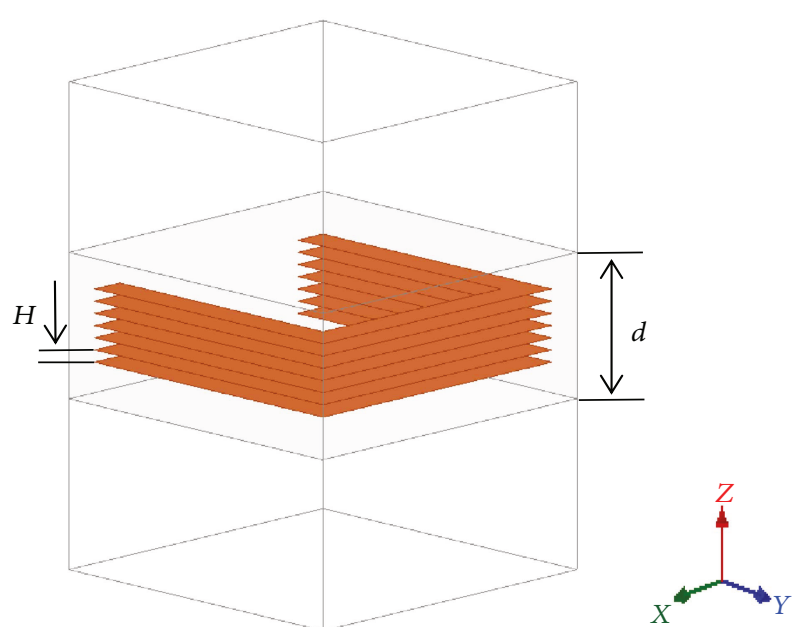

FIgURe 3: Unit cell of ML-UR slab of thickness $d=3 \mathrm{~mm}$, relative permittivity $\varepsilon_{\mathrm{r}}=2.94$, and seven rings along $z$-axis with $H=0.25 \mathrm{~mm}$ spacing. The slab is surrounded by air.

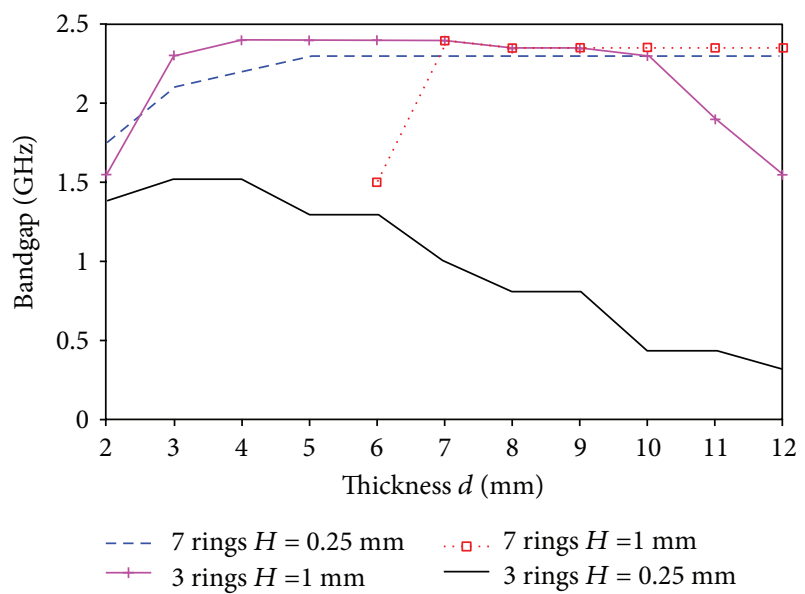

FIGURE 4: Variation of the band gap of ML-UR slabs comprised of 3 and 7 rings along $z$-axis, separated by $0.25 \mathrm{~mm}$ and $1 \mathrm{~mm}$ spacing.

the top and bottom faces of the unit cell. It has been established in [5] that this configuration demonstrates a band gap from $8 \mathrm{GHz}$ to $10.15 \mathrm{GHz}$.

Dispersion characteristics shown in Figure 4 demonstrate the impact of spacing $(H)$ between two adjacent layers along $z$-axis on the band gap. The width of the band gap can be maximized by the selection of optimal number of rings for the given $H$ and slab thickness $d$. A slab of three rings with $0.25 \mathrm{~mm}$ (1 mm) spacing offers a low (high) BG, which decreases with increasing slab thickness $d$. In other words, the increased thickness $d$ of the slab led to the excitation of the guided modes to the detriment of the band gap [1]. Slabs of seven rings with $0.25 \mathrm{~mm}$ and $1 \mathrm{~mm}$ spacing offer considerable constant BG. Therefore, given the minimal difference between BGs of three and seven rings with $1 \mathrm{~mm}$ and $0.25 \mathrm{~mm}$ spacing, respectively, significant fabrication flexibility exists.

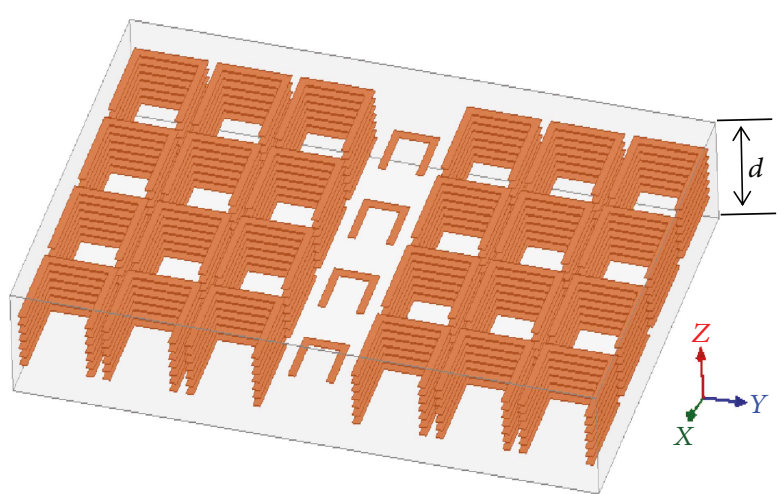

(a)

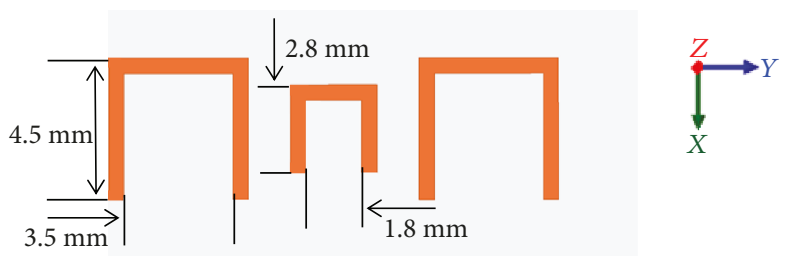

(b)

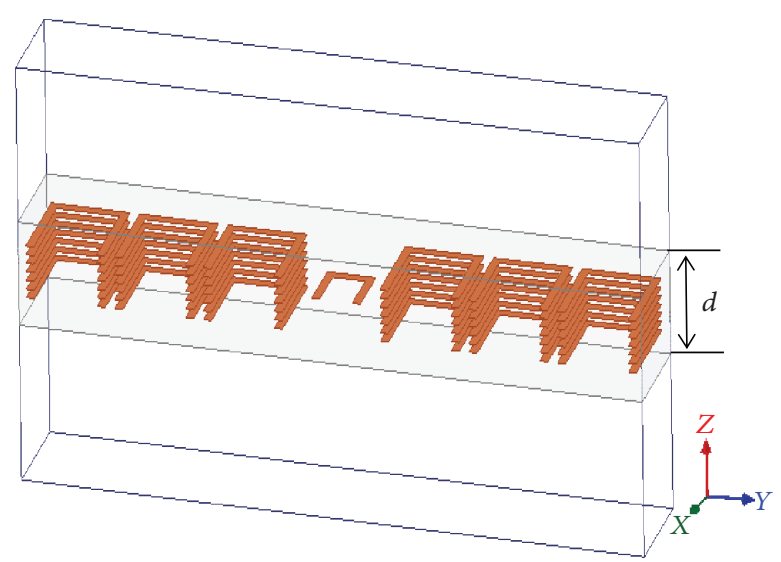

(c)

Figure 5: (a) Schematic diagram of the single-layer line defect slab waveguide. The waveguide has three rows of ML-UR surrounding the channel. (b) Ring dimension. (c) The supercell used to calculate the dispersion relation.

\section{EBG Slab Waveguide}

4.1. Design of Single-Layer Line Defect Waveguide. A waveguide was created within the ML-UR slab by introducing a single-layer channel line defect of reduced size rings in order to confine the field to the defect region. Figure 5(a) illustrates the waveguide configuration, which is a variation of the EBG guide that was recently reported in [5]. The structure was composed of three rows (periods) of ML-UR on either side of the channel as opposed to the single row for the structure that was reported in [5]. The dimension of the UR in the defect region was set to $2.8 \mathrm{~mm}$ as shown in Figure 5(b). In order to calculate the dispersion relation of the structure, the computational supercell [6] of Figure 5(c) was used, with periodic boundary 
conditions on $x$-faces and perfect magnetic conductors on $z$ - and $y$-faces.

Dispersion characteristics of the corresponding guided mode for different numbers of rows on the sides of the channel are shown in Figure 6. It is observed that the number of rows of the perfect crystal on the sides of the channel does not have a significant impact on the guided mode.

A CPW-to-slotline transition (Figure 7) adopted from [7] was used to excite the waveguide. Back-to-back configurations of lengths $L=49 \mathrm{~mm}$ and $69 \mathrm{~mm}$ were designed and fabricated on standard 60 mils RT/Duroid 6002 substrate of $\varepsilon_{r}=2.94$ and copper thickness of 18 microns. The transition was defined by the following parameters: gap width $W_{\mathrm{g}}=0.1 \mathrm{~mm}$, strip width $W_{\mathrm{c}}=1.25 \mathrm{~mm}$, and slot width $W_{\mathrm{s}}=0.7 \mathrm{~mm}$.

Figure 8 depicts the planar structure of the $\mathrm{CPW}$-slotlinefed EBG channel. The feeding transitions and the single-layer line defect of the guide are placed on the same side of the substrate. It is to be noted that the single-layer defect is on the same height as the fourth layer of the full crystal (Figure 5(a)). Layered rings were etched on Duroid 6002 of $0.25 \mathrm{~mm}$ thickness.

The overall dimensions of the waveguide were $L \times W \times H=49 \mathrm{~mm} \times 35 \mathrm{~mm} \times 3 \mathrm{~mm}$ and channel length was set at four periods. Three configurations of the waveguide model with several lengths were designed and fabricated. The waveguide configurations are referred to as waveguide with one [5], three, and six rows of layered rings on the sides of the guiding channel. Figure 9 shows a photograph of the fabricated waveguide configurations with transitions fed by SMA connectors. The left- and right-side numbers of a labeled waveguide represent the number of rows on the sides of the channel and the number of periods along the channel, respectively. Rogers 2929 bonding thin adhesive films with a dielectric constant close to that of Rogers 60 mils substrate were used to assemble the layers.

\section{Waveguide Characterization}

A deembedding technique [8] was applied to the measurements to remove the impact of transitions. The deembedding proposed in [9] is used in this paper. The method is based on bisection of a single two-port THRU structure. It utilizes transmission matrix to split mathematically a symmetric THRU into mirrored halves which is then removed mathematically from both ports of the two ports of measured structure, leaving only the device under test (DUT). This method provides an alternative to open-short deembedding techniques that is restricted by assumptions on the lumped element [10-12]. A brief overview of the deembedding process is presented in here for the sake of completeness.

5.1. Model for Deembedding. The method developed here employs the structures shown in Figure 10 which is composed of the DUT (Figure 10(a) EBG channel) that was placed between symmetric halves of the THRU (back-toback CPW-slotline transition of Figure 10(b)).

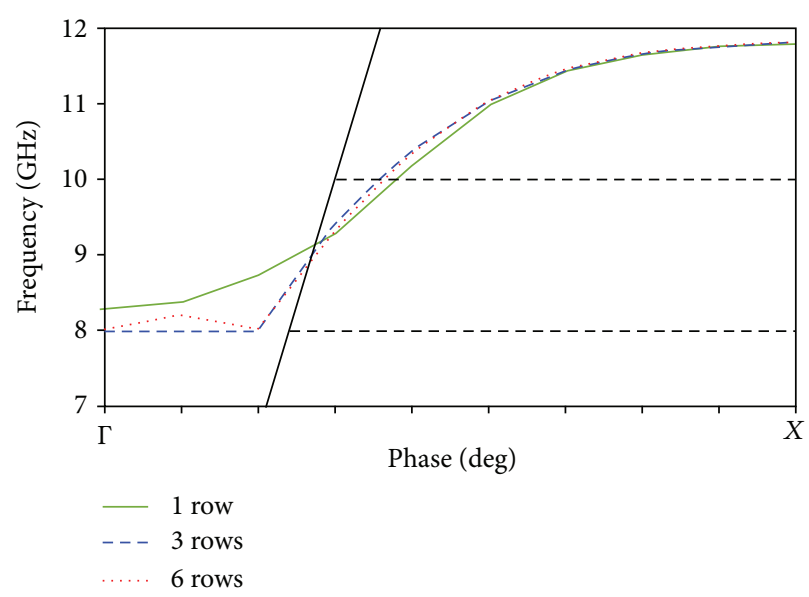

FIGURE 6: Band diagram of guided mode of EBG guide with various numbers of rows on the sides of the channel. The three-row case is shown in Figure 5(c).

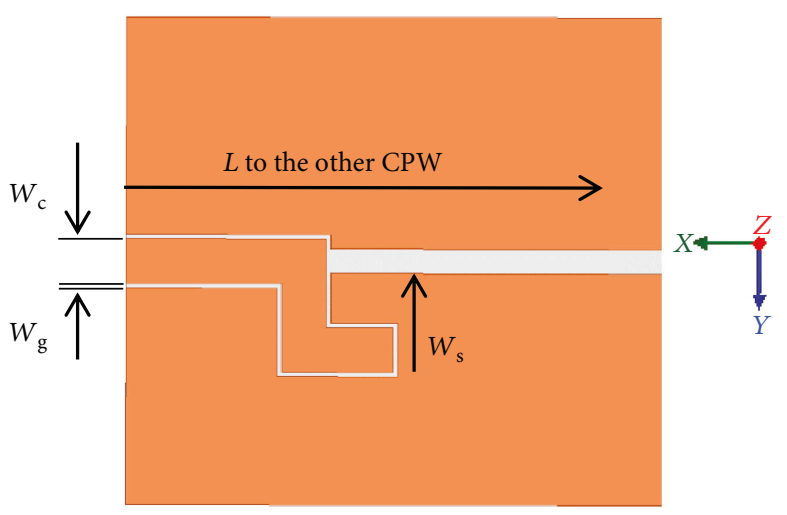

Figure 7: Geometry of the CPW-to-slotline transition.

The THRU was bisected into mirror image halves. This was accomplished by solving the following $\mathrm{ABCD}$ matrix:

$$
\mathbf{A}_{\mathrm{HALF}} \times \overline{\mathbf{A}_{\mathrm{HALF}}}=\mathbf{A}_{\mathrm{THRU}},
$$

where the overbar shows symmetric conjugation defined as follows:

$$
\overline{\left[\begin{array}{ll}
\mathrm{a} & \mathrm{b} \\
\mathrm{c} & \mathrm{d}
\end{array}\right]} \equiv \frac{1}{\mathrm{ad}-\mathrm{bc}}\left[\begin{array}{ll}
\mathrm{d} & \mathrm{b} \\
\mathrm{c} & \mathrm{a}
\end{array}\right] .
$$

Half THRU structure was modeled using HFSS. Based on [13], the simulated model of half structure can be used to deembed the DUT. The following deembedding equation was henceforth used to calculate the ABCD matrix of the DUT from the measured data:

$$
\mathbf{A}_{\mathrm{DUT}}=\left[\mathbf{A}_{\mathrm{HALF}}\right]^{-1} \cdot\left[\mathbf{A}_{\mathrm{MEAS}}\right] \cdot\left[\overline{\mathbf{A}_{\mathrm{HALF}}}\right]^{-1} .
$$

5.2. Results and Discussion. The deembedding method above was applied to measurement of the waveguide configurations of 3-4, 3-8, and 3-16 of Figure 9. The results of the simulated 


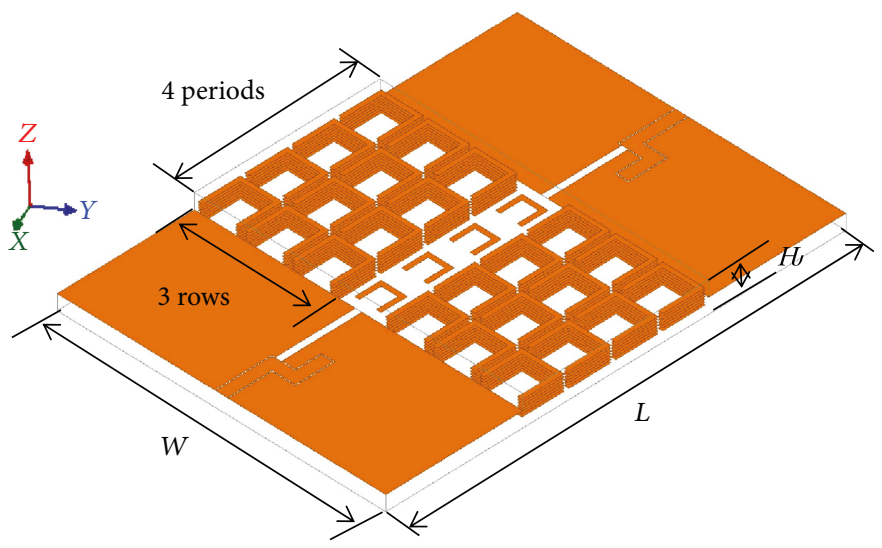

(a)

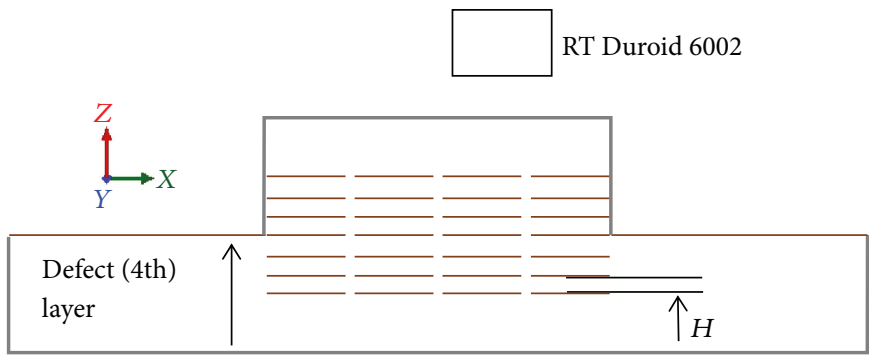

(b)

FIGURE 8: EBG waveguide in dielectric, fed by CPW-to-slotline transitions. (a) Perspective view. (b) Side view. The waveguide has three rows on the sides of the channel and four periods along the channel. The size of the waveguide is $L \times W \times H=49 \mathrm{~mm} \times 35 \mathrm{~mm} \times 3 \mathrm{~mm}$ and layered rings are spaced by $\mathrm{H}=0.25 \mathrm{~mm}$.

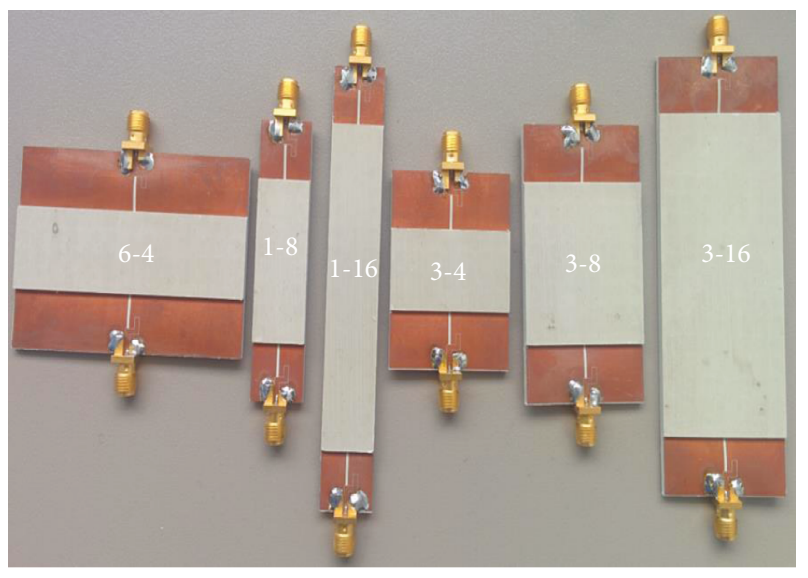

Figure 9: Photo of fabricated line defect waveguides. The left-side number of a labeled waveguide is the number of rows on the sides of the channel while the right-side number is the number of periods along the channel.

and calculated (1) THRU structures $(L=29.2 \mathrm{~mm})$, along with measured back-to-back short transition $(L=49 \mathrm{~mm})$, are plotted in Figure 11 that shows a good agreement between simulated and calculated THRUs from (1). Measured back-to-back transition exhibits some deviation below $10 \mathrm{GHz}$. This discrepancy can be attributed to leakage losses and the connection between SMA connectors and the feed point of the CPW.
Measured results for performance of the EBG guide of different numbers of rows on the sides of the channel, and also, channel lengths are shown in Figures 12, 13, and 14 before and after the deembedding process. The simulated results (Raw) of the device under test (DUT) are also plotted for comparison with the deembedded results. Deembedded return loss is better than $11 \mathrm{~dB}$ in the $9.5-11.1 \mathrm{GHz}$ range. Deembedded insertion loss has a mean value of $3 \mathrm{~dB}$, $3.3 \mathrm{~dB}$, and $4.3 \mathrm{~dB}$, as compared to the simulated mean value of $2.4 \mathrm{~dB}, 2.5 \mathrm{~dB}$ and $2.8 \mathrm{~dB}$ within the same frequency band for the 4-period, 8-period, and 16-period guides, respectively. Note that increasing the length of an EBG-channel does not degrade the insertion loss performance of the waveguide as expected but causes a marginal improvement, which is an indication of the impact of the transition on the EBG guide. It can be seen that there is a good agreement between deembedded results and simulated performance for the majority of the fabricated prototypes. Deviations between simulated and deembedded results can be attributed to the manufacturing imperfections, soldering of the connectors and CPW feed line, and conductor losses. Table 2 summarizes the performance of the fabricated deembedded EBG waveguide prototypes in the frequency range $9.5-11 \mathrm{GHz}$.

Eigen-mode simulations of Figure 6 showed that the number of rows on the sides of the channel has a marginal impact on the dispersion of the guided mode. However, Table 2 shows otherwise. Indeed, the insertion loss has been increased by increasing the number of rows on the sides of 


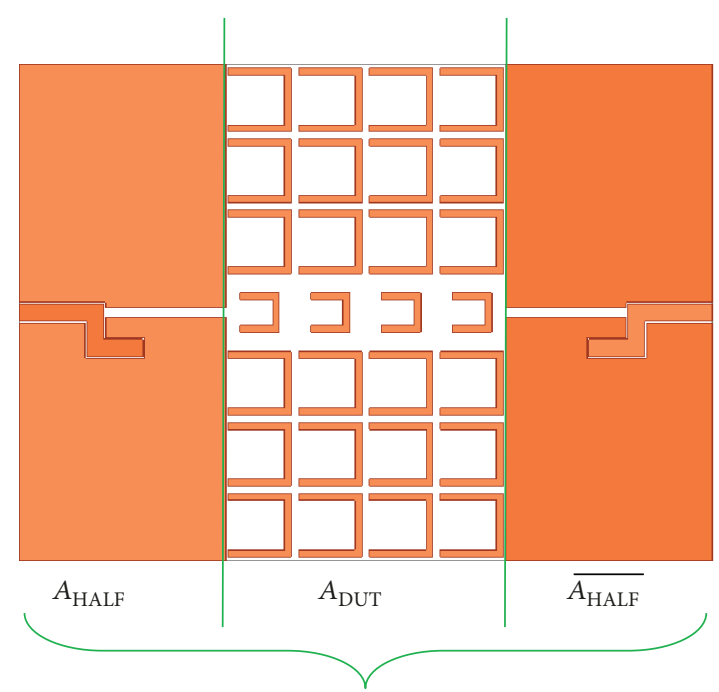

(a)

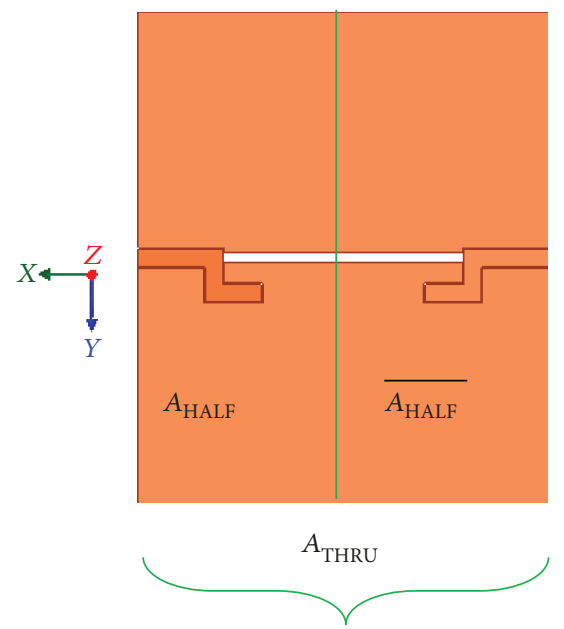

(b)

FIGURE 10: Deembedding technique using transmission matrix bisect deembedding. (a) Measured structure having the DUT. (b) THRU structure.

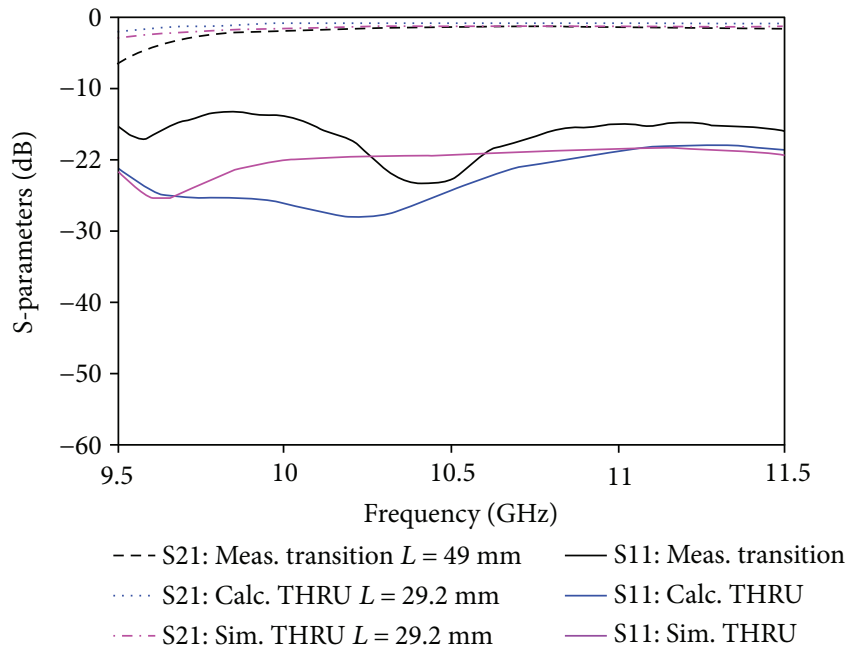

FIGURE 11: S-parameters of the calculated, simulated THRU structure of length $L=29.2 \mathrm{~mm}$ and measured back-to-back transition of length $L=49 \mathrm{~mm}$.

the channel for the same length of the channel. This is contrary to what was observed in the study of the foamsupported channel [4]. This might stem from excitation of surface waves for the dielectric structure as compared to the EBG waveguide with foam spacer. Waveguides with three and six rows and channel length of four periods show $3 \mathrm{~dB}$ and $5.4 \mathrm{~dB}$ losses, respectively. Meanwhile, waveguides with one and three row(s) and channel length of eight periods offer insertion losses of $2.6 \mathrm{~dB}$ and $3.3 \mathrm{~dB}$, respectively. In other words, surface modes are more pronounced for EBG guides with larger numbers of rings on the sides of the channel [14] as can be seen in Figures 15(b)-15(d). These guided modes couple to the surface mode at the output port of the channel, which results in loss. It is important to note that the loss due to increased channel length is smaller than that due to increased side rows. Specifically, in the case of waveguide with three rows and channel length of four periods, adding rows along the guiding direction introduces marginal increase in the loss $(1.3 \mathrm{~dB}$ for the longer sixteen period channel) as compared to the case when rows on the sides of the channel are widened leading to appreciable loss ( $2.4 \mathrm{~dB}$ for the thicker six-row channel). This is due to the effect of distributed Bragg reflection (DBR) [15] along the guiding direction and stronger interaction region between the propagating mode and the surrounding crystal. The effect of the DBR does not change significantly with the length of the waveguide. This argument reveals why increasing the length of the channel has marginally impacted the loss of the waveguide. However, the coupling to the surface modes is enhanced as the row EBG around the channel is widened. The longer sixteen-period guide with one row on each side of the channel exhibited the lowest loss, $0.35 \mathrm{~dB} / \mathrm{cm}$. This can be explained by noting that local surface waves are weaker (thinner channel) as shown in Figure 15(a).

Note that decreasing the number of rings along $z$-axis for the same slab thickness $d$, that is, three rings spaced by a $1 \mathrm{~mm}$ case, increases the insertion loss. This is due to the lack of vertical confinement in the layered waveguide [2]. The choice of seven-ring structure ensures the minimum insertion losses with reasonable manufacturing tolerances.

\section{Conclusion}

Detailed dispersion characteristics of a dielectric-based multilayer-U-ring EBG structure were demonstrated for the first time. This structure has two band gaps and a planar configuration. A band gap in the X-band was employed to design single-layer line defect slab waveguides, which allowed single-mode propagation. Multiple waveguides were developed with integrated feeding circuits. Experimental 


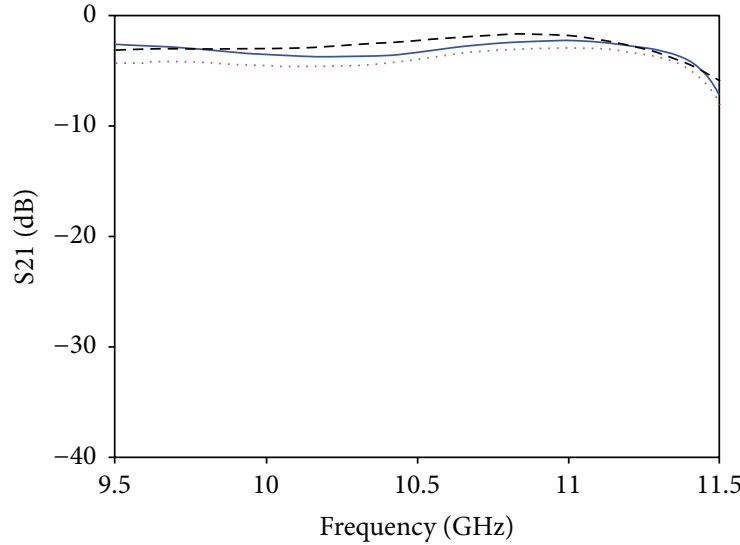

Measured

- Deembedded

--- Raw

(a)

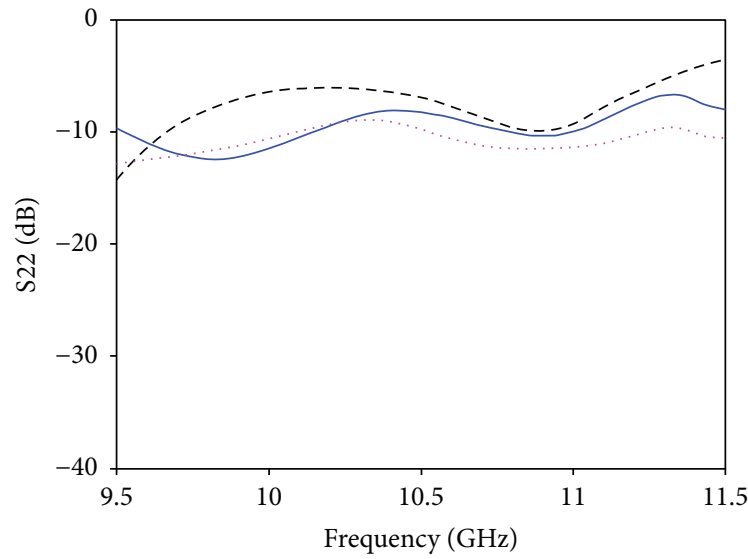

Measured

- Deembedded

-.- Raw

(b)

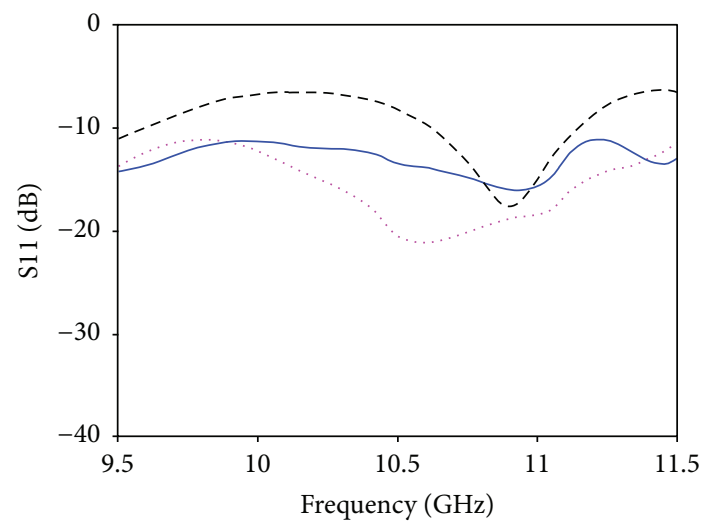

Measured

Deembedded

- - Raw

(c)

FIGURE 12: Characteristic S-parameters before and after deembedding of EBG guide with 3 rows and 4 periods. Raw is the simulated result of DUT. (a) S21, (b) S22, and (c) S11.

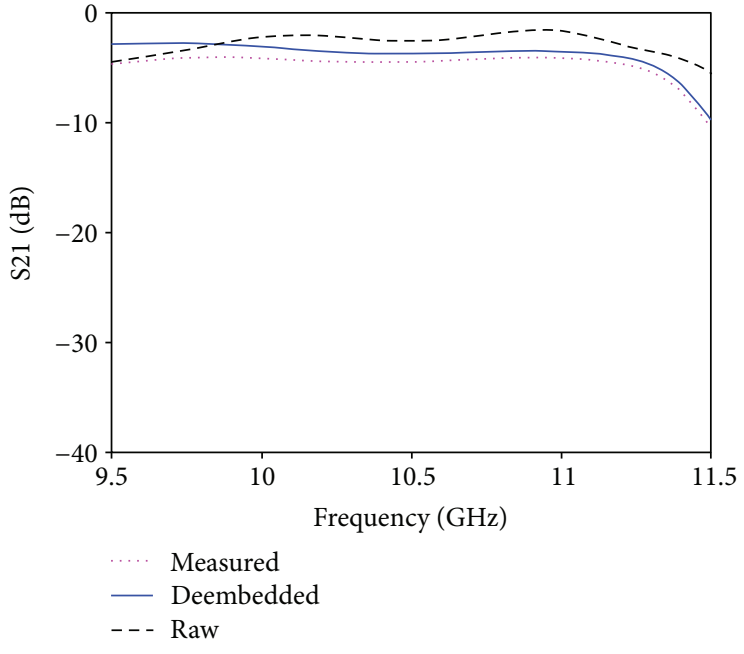

(a)

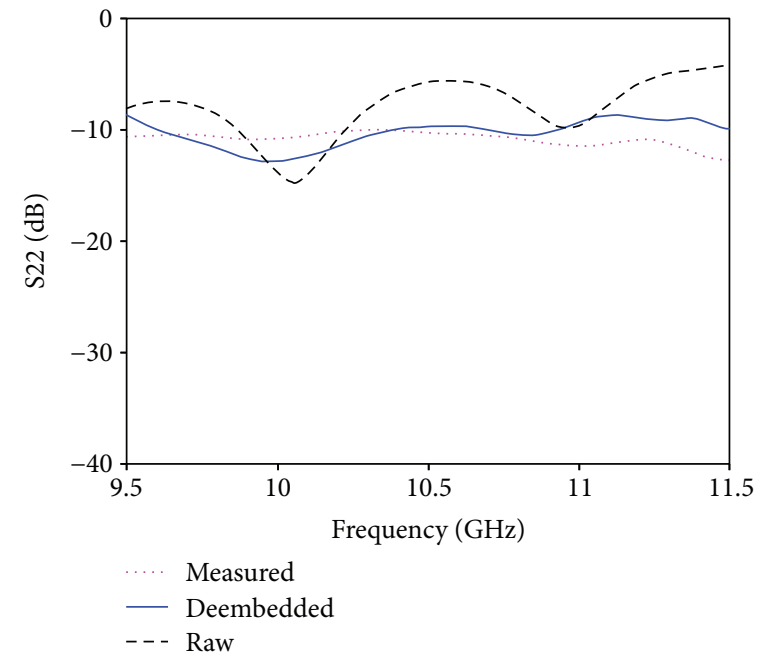

(b)

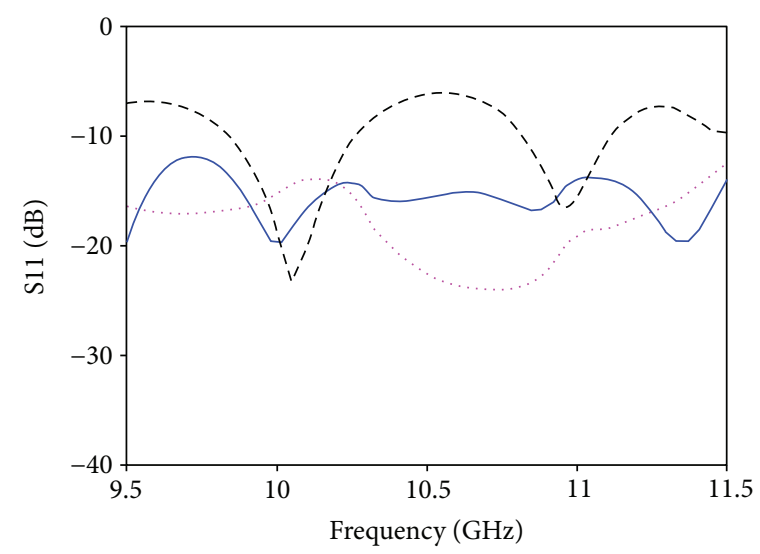

.... Measured

Deembedded

- - Raw

(c)

FIgURE 13: Characteristic S-parameters before and after deembedding of EBG guide with 3 rows and 8 periods. Raw is the simulated result of DUT. (a) S21, (b) S22, and (c) S11. 


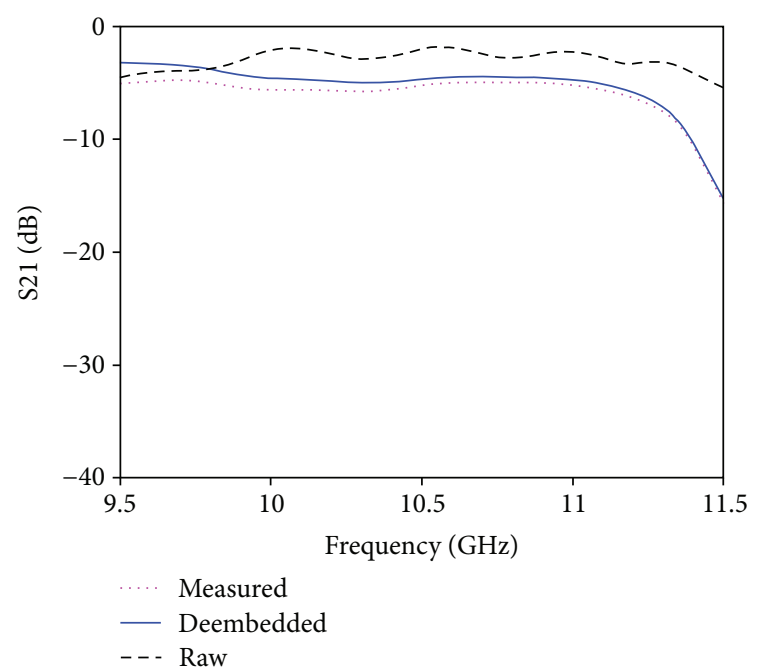

(a)

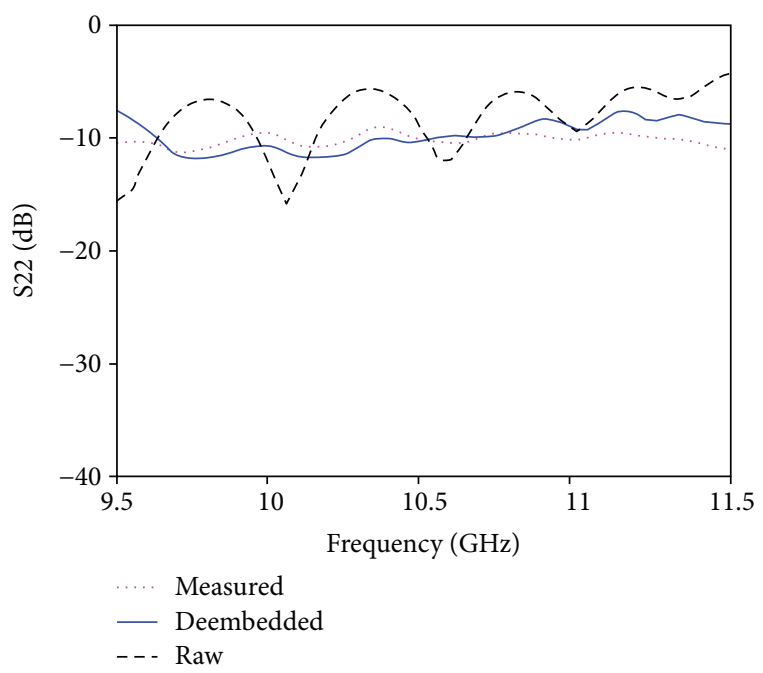

(b)

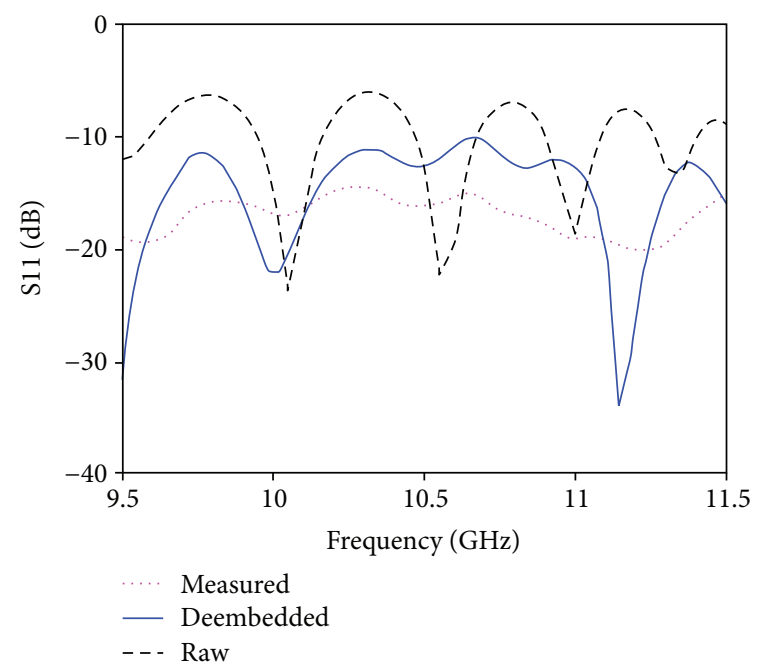

(c)

Figure 14: Characteristic S-parameters before and after deembedding of EBG guide with 3 rows and 16 periods. Raw is the simulated result of DUT. (a) S21, (b) S22, and (c) S11.
Table 2: Performance of the fabricated deembedded waveguides.

\begin{tabular}{lccc}
\hline $\begin{array}{l}\text { Number } \\
\text { of rows }\end{array}$ & $\begin{array}{c}\text { Channel length } \\
\text { (periods) }\end{array}$ & $\begin{array}{c}\text { Return loss } \\
(\mathrm{dB})\end{array}$ & $\begin{array}{c}\text { Mean in band } \\
\text { insertion loss }(\mathrm{dB})\end{array}$ \\
\hline 1 & 8 & $>11$ & 2.6 \\
\hline & 16 & $>11$ & 3.3 \\
3 & 4 & $>11$ & 3 \\
& 8 & $>11$ & 3.3 \\
\hline 6 & 16 & $>11$ & 4.3 \\
\hline
\end{tabular}

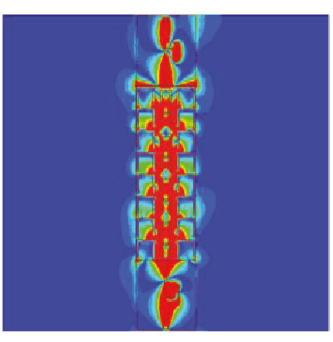

(a)

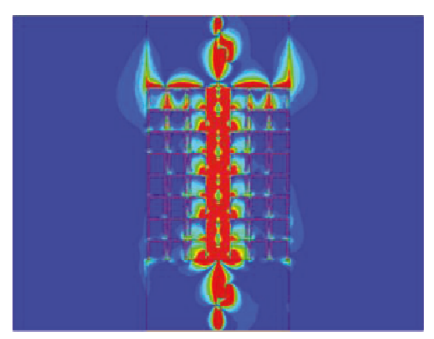

(b)

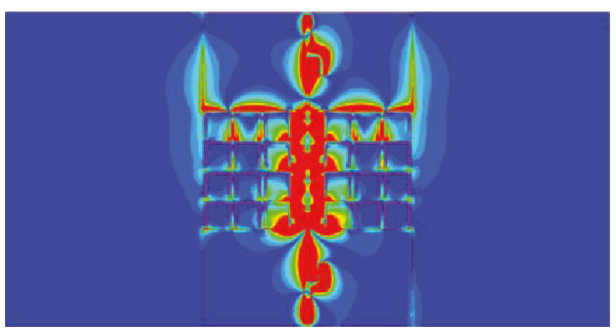

(c)

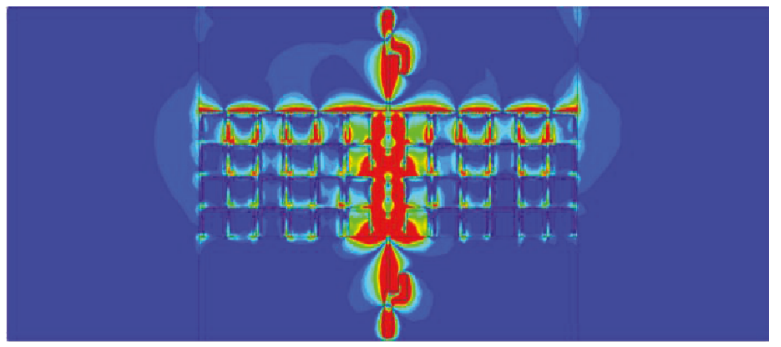

(d)

FIgURe 15: Electric field distribution at $f=10.5 \mathrm{GHz}$ in the EBG waveguide. (a) Channel length of 8 periods with 1 row and (b) with 3 rows. (c) Channel length of 4 periods with 3 rows and (d) and 6 rows.

measurements were consistent with simulation results. The insertion loss decreased by decreasing the number of rows on the sides of the channel for the same length of channel. Despite the imperfections in the fabrication process, measured insertion loss is $0.35 \mathrm{~dB} / \mathrm{cm}$. The insertion loss did not change significantly by increasing the length of the channel which is an indication of the low loss of the EBG guide structure. The suggested EBG has potential applications in antennas, feed distribution networks, and couplers [16]. 


\section{Data Availability}

The data used to support the findings of this study are available from the corresponding author upon request.

\section{Conflicts of Interest}

The authors claim no conflicts of interest.

\section{Acknowledgments}

This work was supported by the Natural Sciences and Engineering Research Council of Canada (NSERC). The authors gratefully acknowledge the support of N. Mikhail, Department of Electronics, Carleton University, Ottawa, ON, Canada, in the computational phase of this work. They would also like to acknowledge Candor Industries, Toronto, ON, Canada, for fabrication of prototypes.

\section{References}

[1] H. Ouassal, J. Shaker, L. Roy, and R. Chaharmir, "A novel multi-layer electromagnetic band gap structure (EBG) comprised of 3-D lattice of square rings," in 2015 IEEE MTT-S International Conference on Numerical Electromagnetic and Multiphysics Modeling and Optimization (NEMO), pp. 1-4, Ottawa, ON, Canada, August 2015.

[2] H. Ouassal, L. Roy, J. Shaker, and R. Chaharmir, "Guided modes of a line-defect EBG slab waveguide in a 3-D square lattice of metallic open square rings," in 2015 Global Symposium on Millimeter Waves (GSMM), pp. 1-3, Montreal, QC, Canada, May 2015.

[3] J. Joannopoulos, R. Meade, and J. Winn, Photonic Crystals: Molding the Flow of Light, Princeton University Press, Princeton, NJ, USA, 1995.

[4] H. Ouassal, J. Shaker, L. Roy, M. R. Chaharmir, and K. Hettak, "A novel multilayer electromagnetic bandgap structure composed of square rings as microwave guiding structures," IEEE Transactions on Components, Packaging and Manufacturing Technology, vol. 7, no. 1, pp. 88-97, 2017.

[5] H. Ouassal, J. Shaker, K. Hettak, R. Chaharmir, and L. Roy, "Compact EBG guides using substrate layered rings," in 2016 17th International Symposium on Antenna Technology and Applied Electromagnetics (ANTEM), pp. 1-3, Montreal, QC, Canada, July 2016.

[6] S. G. Johnson, S. Fan, P. R. Villeneuve, J. D. Joannopoulos, and L. A. Kolodziejski, "Guided modes in photonic crystal slabs," Physical Review B, vol. 60, no. 8, pp. 5751-5758, 1999.

[7] T. G. Lim, H. N. Ang, I. D. Robertson, and B. L. Weiss, "Ka-band CPW-to-slotline transition in photoimageable thick film technology for tapered slot antenna applications," Microwave and Optical Technology Letters, vol. 48, no. 10, pp. 2032-2034, 2006.

[8] R. F. Bauer and P. Penfield, "De-embedding and unterminating," IEEE Transactions on Microwave Theory and Techniques, vol. 22, no. 3, pp. 282-288, 1974.

[9] E. S. Daniel, N. E. Harff, V. Sokolov, S. M. Schreiber, and B. K. Gilbert, "Network analyzer measurement de-embedding utilizing a distributed transmission matrix bisection of a single THRU structure," in ARFTG 63rd Conference, pp. 61-68, Fort Worth, TX, USA, June 2004.
[10] L. F. Tiemeijer and R. J. Havens, "A calibrated lumped element de-embedding technique for on-wafer RF characterization of high-quality inductors and high-speed transistors," IEEE Transactions on Electron Devices, vol. 50, no. 3, pp. 822829, 2003.

[11] M. C. A. M. Koolen, J. A. M. Geelen, and M. P. J. G. Versleijen, "An improved de-embedding technique for on-wafer highfrequency characterization," in Proceedings of the 1991 Bipolar Circuits and Technology Meeting, pp. 188-191, Minneapolis, MN, USA, September 1991.

[12] H. Cho and D. E. Burk, "A three-step method for the deembedding of high-frequency S-parameter measurements," IEEE Transactions on Electron Devices, vol. 38, no. 6, pp. 1371-1375, 1991.

[13] A. Munir, V. Fusco, and O. Malyuskin, "Parallel plate waveguide simulator design, characterisation and DUT deembedding," in 2008 IEEE Antennas and Propagation Society International Symposium, pp. 1-4, San Diego, CA, USA, July 2008.

[14] A. Adibi, Yong Xu, R. K. Lee, A. Yariv, and A. Scherer, "Properties of the slab modes in photonic crystal optical waveguides," Journal of Lightwave Technology, vol. 18, no. 11, pp. 1554-1564, 2000.

[15] S. K. Reddy, A. Adibi, Y. Xu, and R. K. Lee, "Design of electromagnetic modes in photonic crystal optical waveguides," in Proceedings Volume 4655, Photonic Bandgap Materials and Devices, pp. 73-80, San Jose, CA, USA, April 2002.

[16] H. Ouassal, J. Shaker, L. Roy, K. Hettak, and R. E. Amaya, "A novel multifunctional EBG-based coupled-line-defect directional coupler based on layered dielectric substrate," IEEE Transactions on Components, Packaging and Manufacturing Technology, pp. 1-12, 2018. 


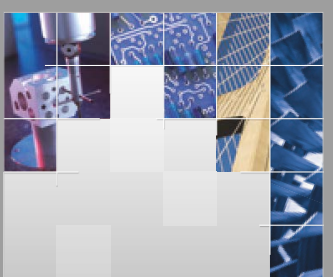

\section{Enfincering}
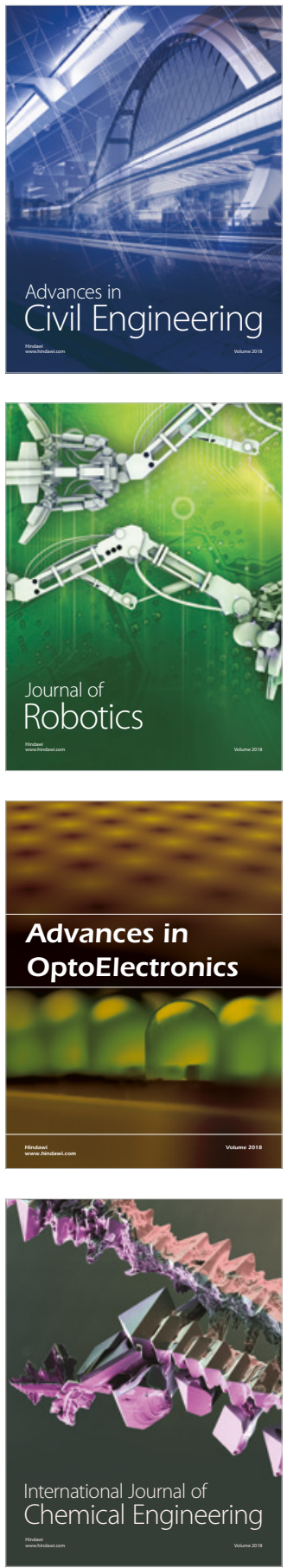

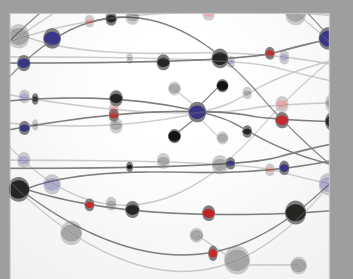

\section{Rotating \\ Machinery}

The Scientific World Journal

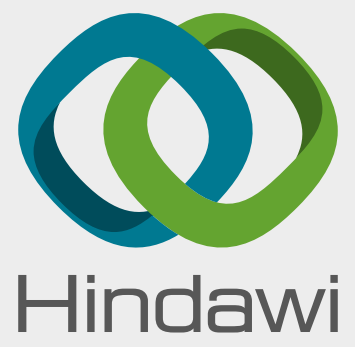

Submit your manuscripts at

www.hindawi.com
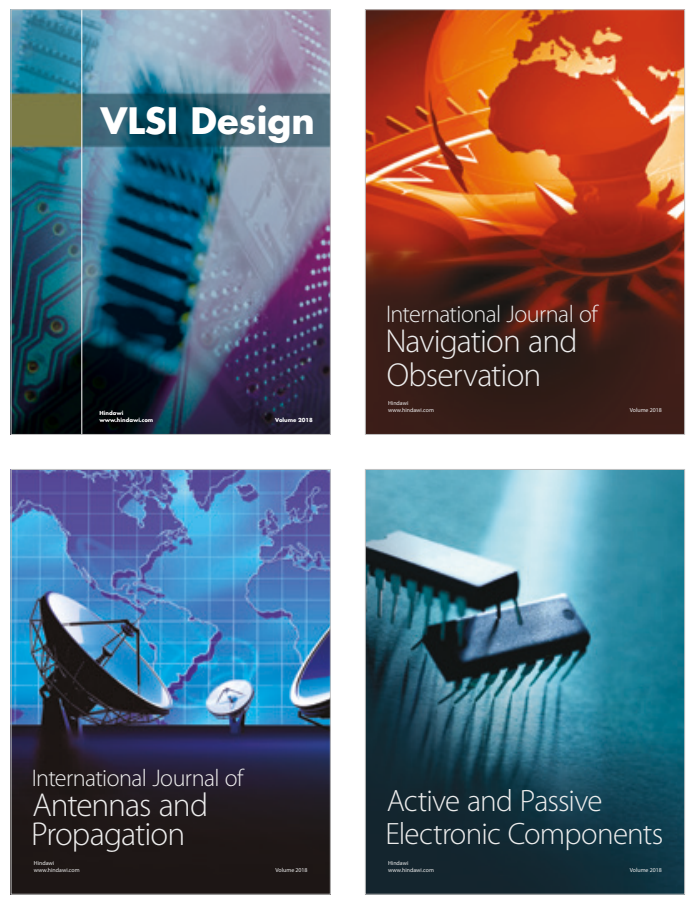
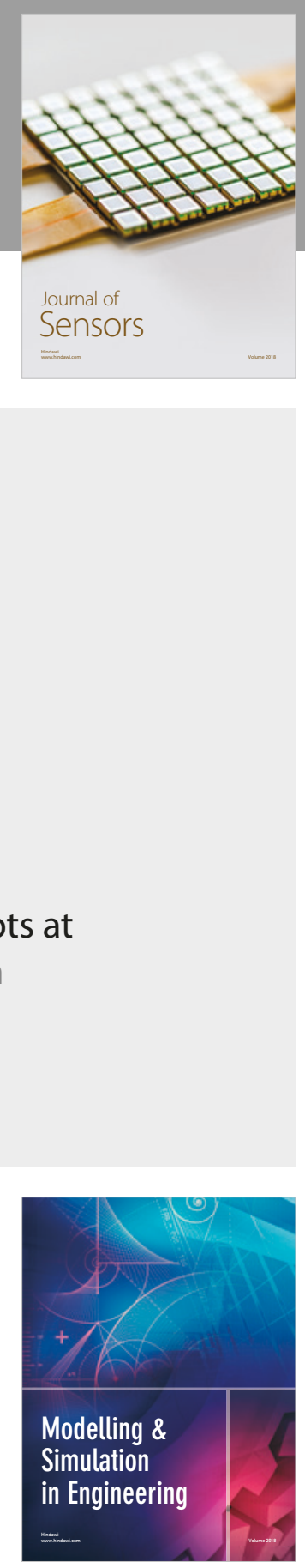

\section{Advances \\ Multimedia}
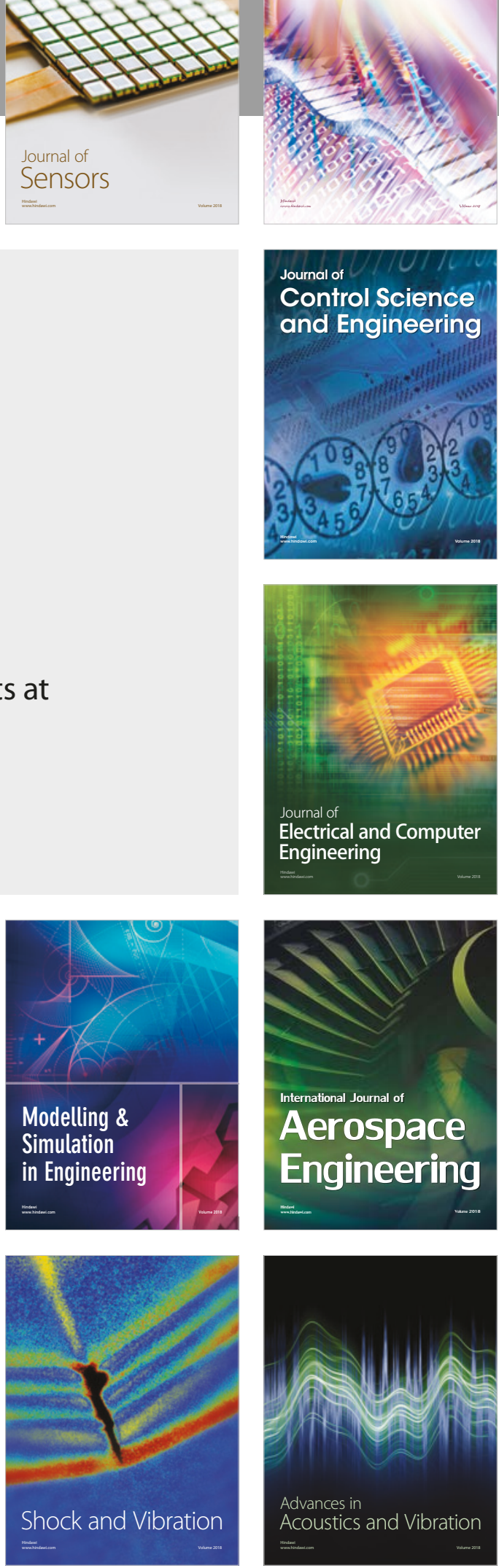